\title{
Size effect in the strength of concrete structures
}

\author{
B L KARIHALOO and Q Z XIAO \\ Division of Civil Engineering, School of Engineering, Cardiff University, \\ PO Box 925, Queen's Buildings, Cardiff CF24 0YF, UK \\ e-mail: karihalooB@ cardiff.ac.uk
}

\begin{abstract}
This paper reports on the range of applicability of the various size effect formulae available in the literature. In particular, the failure loads of three point bend (TPB) beams are analysed according to the size effect formulae of Bažant and of Karihaloo for notched beams and according to those of Bažant and of Carpinteri for unnotched beams, and the results of this analysis presented. Improvements to Karihaloo's size effect formula are also proposed.
\end{abstract}

Keywords. Fictitious crack model; fracture process zone (FPZ); high strength concrete (HSC); size effect; three point bend (TPB) beam.

\section{Introduction}

The fracture mechanics size effect, as opposed to the Weibull statistical size effect, is a controversial topic in the fracture of concrete. Yet, it is fracture mechanics alone that can illuminate the hitherto unexplained size effect observed in the strength of concrete structures. It would therefore seem appropriate to conduct dedicated experiments in order to establish the range of applicability of the several fracture mechanical size effect formulae available in the literature (Bažant 1984, 1997; Carpinteri 1994a; Karihaloo 1999).

Bažant (1984), using the energy release rate concept, proposed the formula,

$$
\left(\sigma_{N}\right)_{u}=A_{1}\left[1+\left(B_{1} / W\right)\right]^{-1 / 2},
$$

where $\left(\sigma_{N}\right)_{u}$ is the nominal stress at failure of a structure of specified shape and loading condition, $W$ is a characteristic size of the structure, and $A_{1}$ and $B_{1}$ are positive constants. These depend on the fracture parameters, $G_{f}$ and $c_{f}$, of concrete and on the notch to depth ratio. $G_{f}$ represents the specific fracture energy and $c_{f}$ the corresponding fracture process zone (FPZ) length of an infinitely large specimen. They are determined by regression analysis of tests on geometrically similar notched specimens. Bažant's formula reduces to the linear elastic fracture mechanics (LEFM) limit as $W \rightarrow \infty$. In fact, (1) has been established by Taylor's expansion from this asymptotic limit (Karihaloo 1995).

Karihaloo (1999), using the stress intensity factor and the fictitious crack concepts, proposed the formula

$$
\left(\sigma_{N}\right)_{u}=A_{2}\left[1-\left(B_{2} / W\right)\right]^{1 / 2},
$$


where $A_{2}$ and $B_{2}$ are constants. These constants also depend on the fracture parameters $G_{F}$ and $\ell_{p}$ of concrete and on the notch to depth ratio. Note that $G_{F}$ and $\ell_{p}$ are consistent with the Hillerborg fictitious crack model. They differ from $G_{f}$ and $c_{f}$ in (1). Equation (2) also reduces to the LEFM limit as $W \rightarrow \infty$. It is, however, unlikely to be applicable when $W$ is small, which is a consequence of several approximations and assumptions made in its derivation. These will be discussed later.

Many quasi-brittle structures are known to fail at crack initiation, although the process zone is well developed. For such structures the nominal failure stress was found to approach the LEFM limit for $W \rightarrow \infty$ from the above,

$$
\left(\sigma_{N}\right)_{u}=\left[A_{3}+\left(B_{3} / W\right)\right]^{1 / 2},
$$

Here, $A_{3}$ and $B_{3}$ are positive constants which are determined by regression analyses of tests on geometrically similar unnotched beams. This formula was obtained by Carpinteri and his co-workers using multifractal scaling concepts (Carpinteri 1994; Carpinteri \& Ferro 1994). Bažant (1997) also obtained a size effect formula for initially-unnotched structures using the same concepts as for the notched structures. His formula, however, differs from (3) in that the exponent is approximately 0.7 .

The objectives of this paper are two-fold. First, it compares the size effect formulae, (1) and (2), for tests on high strength concrete (HSC, nominal compressive strength $110 \mathrm{MPa}$ ) with a view to identifying their ranges of applicability. Beams (span to depths ratio of 4) with depths varying between 50 and $400 \mathrm{~mm}$ with a central edge notch were tested in three point bending (Karihaloo \& Abdalla 2001). The notches ranged in depth from the very shallow (notch to depth ratio 0.05) to the deep (notch to depth ratio 0.3). Comparison is also made of the size effect formulae for unnotched HSC beams ranging in depth between 50 and $400 \mathrm{~mm}$.

The paper also attempts to eliminate most of the assumptions made in the derivation of (2) with a view to obtaining better understanding of the stress redistribution in the FPZ and of its role in the size effect of concrete structures.

\section{Notched HSC beams}

Tests were conducted by Karihaloo \& Abdalla (2001) on notched HSC beams (span to depth ratio $=4$, width $B=100 \mathrm{~mm}$ ) in three point bending. The mean values (and coefficients of variation) of the measured mechanical properties are: compressive strength $108.8 \mathrm{MPa}$ $(3.2 \%)$, split cylinder strength $7.40 \mathrm{MPa}(4.9 \%)$, modulus of elasticity $40.45 \mathrm{GPa}(3.9 \%)$, and specific fracture energy $44.7 \mathrm{~J} / \mathrm{m}^{2}(8.7 \%)$.

Three beams were tested for each of the three notch to depth ratios $(\alpha=0.05,0.10,0.30)$. For the smallest notch to depth ratio only two beam depths were tested. The mean values of the nominal failure strength are given in table 1. The nominal failure strengths are plotted in figures 1-3 for the three notch depths and compared according to (1) and (2).

From the analysis presented in figures 1-3, the following conclusions can be drawn.

- For notched HSC beams with notch to depth ratios of 0.05 and 0.10 , the predictions of both (1) and (2) deviate somewhat from the measured nominal strengths. The deviation of (2) for small sizes is particularly evident. The possible reasons for this will be discussed below.

- For notched HSC beams with notch to depth ratio of 0.3 or more, the predictions of both formulae are in good agreement with measured values for large sizes. However, somewhat surprisingly, (2) alone appears to predict the correct trend for small sizes. 
Table 1. Failure loads for HSC beams.

\begin{tabular}{lccc}
\hline $\begin{array}{l}\mathrm{W} \\
(\mathrm{mm})\end{array}$ & $\alpha$ & $\begin{array}{c}\text { Mean } \\
P_{u}(\mathrm{kN})\end{array}$ & $\begin{array}{c}\text { Mean } \\
\left(\sigma_{N}\right)_{u}(\mathrm{MPa})\end{array}$ \\
\hline 200 & 0.05 & 22.79 & 1.14 \\
400 & & 36.06 & 0.90 \\
100 & & 10.88 & 1.09 \\
200 & 0.10 & 17.67 & 0.88 \\
400 & & 27.70 & 0.69 \\
75 & & 4.75 & 0.63 \\
150 & 0.30 & 8.17 & 0.54 \\
300 & & 12.66 & 0.42 \\
\hline
\end{tabular}

\section{Improvement of (2)}

In the derivation of (2) (Karihaloo 1999), it was recognised that quasi-brittle materials develop a diffuse FPZ before the formation of a traction-free crack whose size can be commensurate with that of a small test specimen. Within this zone the stresses are redistributed so that it is necessary to consider not only the singular term in the asymptotic crack tip field but also higher

(a)

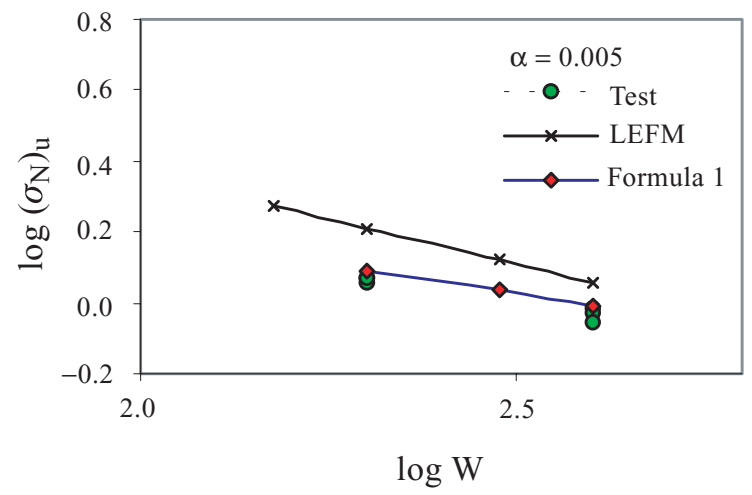

(b)

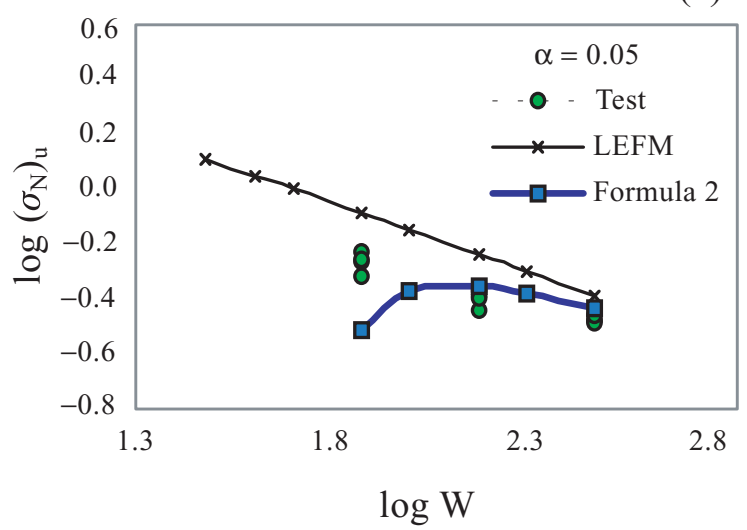

Figure 1. Size effect plot for HSC specimens with $\alpha=0.05$ according to (1) (a) and (2) (b). 
(a)

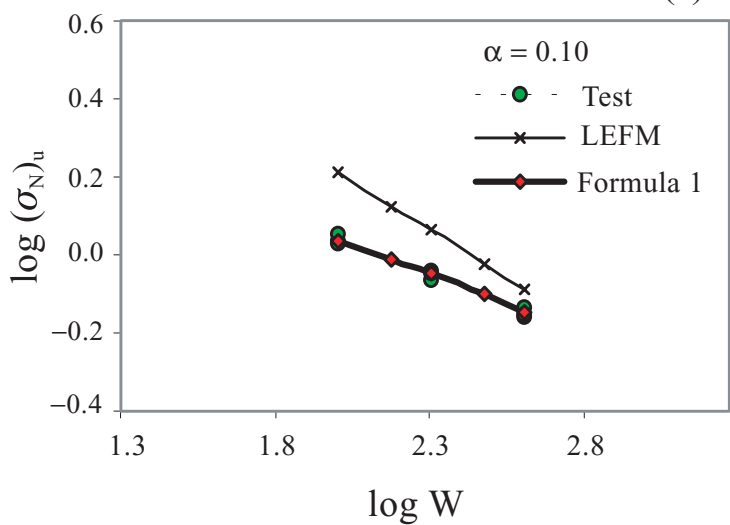

(b)

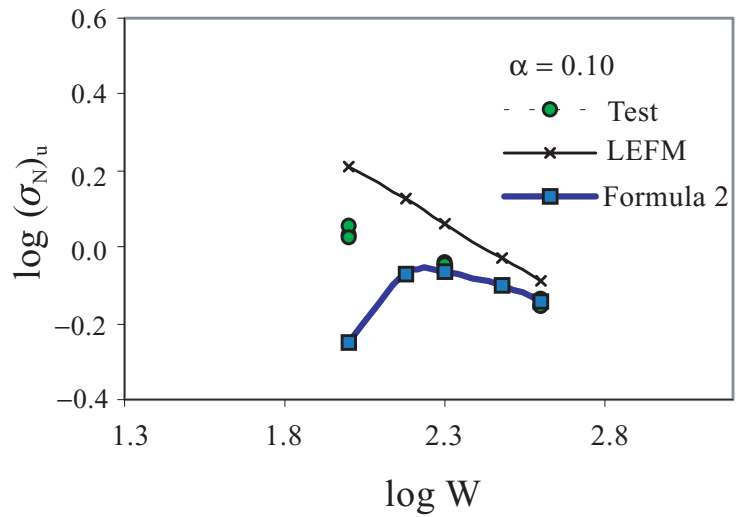

Figure 2. Size effect plot for HSC specimens with $\alpha=0.10$ according to (1) (a) and (2) (b).

order, nonsingular terms. In the derivation proper, Karihaloo (1999) used approximations for the higher order terms, as well as the weight (Green's) functions for a semi-infinite crack in an infinite plane instead of a finite size crack in a finite TPB specimen. These approximations were made in order to arrive at the close form expression for nominal strength (2). They are pointed out below when the exact formulation is introduced. The approximations have been recently eliminated by taking into account accurate higher order terms of the crack tip asymptotic field, as well as by using the weight functions for a finite crack in a finite TPB specimen.

In common with the earlier derivation (Karihaloo 1999), the traction-free crack with a FPZ of length $\ell_{p}$ at its tip is decomposed into a traction-free crack (figure 4) with the following stress field at its tip

$$
\sigma_{y}(r) \equiv \sigma_{o}(r)=\frac{a_{1}}{\sqrt{r}}+3 a_{3} \sqrt{r}+5 a_{5} r^{3 / 2},
$$

and the FPZ with the stress $\left[\sigma(s)-\sigma_{o}\left(\ell_{p}-s\right)\right]$ and the displacement $w(s)$ across its faces. In (4), $a_{1}$ is related to the mode I stress intensity factor (SIF) $K_{I}$ via $a_{1}=K_{I} / \sqrt{2 \pi}$. The coefficients $a_{1}, a_{3}$ and $a_{5}$ depend on the crack length, applied load $\sigma_{r}$ and size and geometry 
(a)

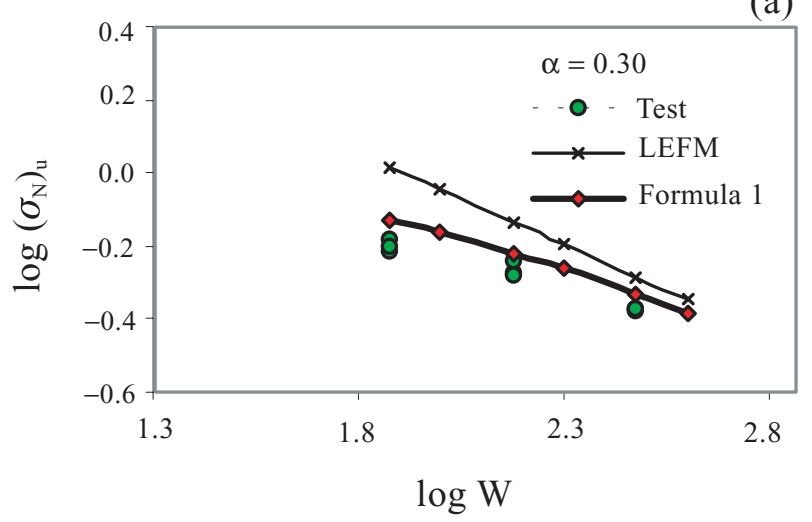

(b)

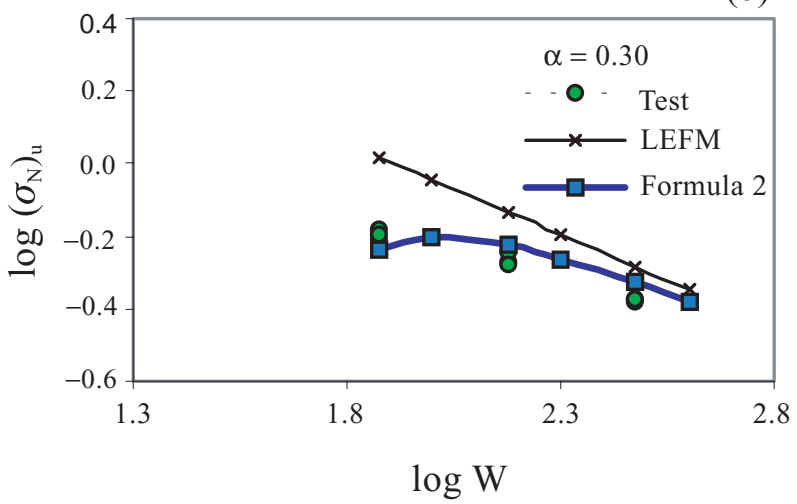

Figure 3. Size effect plot for HSC specimens with $\alpha=0.30$ according to (1) (a) and (2) (b).

of the body. Solutions for TPB beam and a typical wedge-splitting geometry have been recently obtained by Karihaloo \& Xiao (2001). In the previous approximate formulation (Karihaloo 1999), only the coefficients $a_{1}$ and $a_{3}$ were retained. Moreover, $a_{3}$ was obtained in an approximate manner.

For a TPB beam with a span to depth ratio of 4 , the coefficients $a_{1}, a_{3}$, and $a_{5}$ are (with $\alpha=$ notch to depth ratio),

$$
\begin{aligned}
& a_{1}=\sigma_{r} \sqrt{W} k_{4}(\alpha), \\
& a_{3}=\left(\sigma_{r} / \sqrt{W}\right) g_{4}^{3}(\alpha), \\
& a_{5}=\left(\sigma_{r} / W^{3 / 2}\right) g_{4}^{5}(\alpha)
\end{aligned}
$$

where

$$
\begin{aligned}
k_{4}(\alpha) & =\sqrt{\alpha} p_{4}(\alpha) /\left[\sqrt{2 \pi}(1-\alpha)^{3 / 2}(1+3 \alpha)\right], \\
p_{4}(\alpha) & =1.9+0.41 \alpha+0.51 \alpha^{2}-0.17 \alpha^{3}, \\
g_{4}^{3}(\alpha) & =0.6534-9.2406 \alpha+49.515 \alpha^{2}-153.97 \alpha^{3}+233.48 \alpha^{4}-148.73 \alpha^{5}, \\
g_{4}^{5}(\alpha)= & 2.1491-52.998 \alpha+468.48 \alpha^{2}-2084.4 \alpha^{3}+4919.3 \alpha^{4} \\
& -5869.4 \alpha^{5}+2765.2 \alpha^{6} .
\end{aligned}
$$


(a)
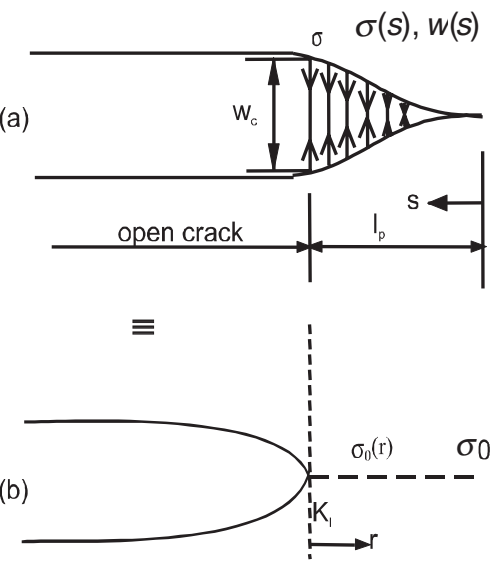

(c)

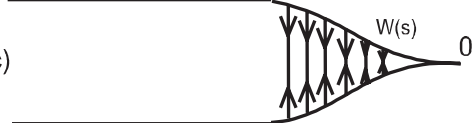

(d)

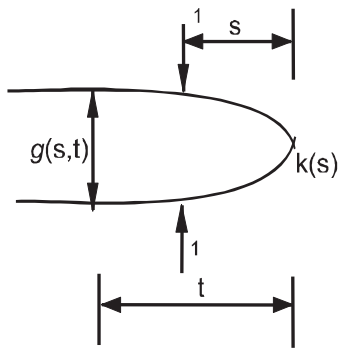

Figure 4. Decomposition of a traction free crack with a fracture process zone of length $l_{p}(\mathbf{a})$ into the traction free crack with stress $\sigma_{0}(r)$ ahead of crack tip (b) and the FPZ with stress $\left[\sigma(s)-\sigma_{0}\left(l_{p}-s\right)\right]$ and displacement $w(s)$ (c). As the faces close smoothly the stress intensity factor at 0 will vanish. $k(s)$ is the stress intensity factor due to unit concentrated loads at $s, g(s, t)$ is the corresponding displacement at location $t$ (d). (From Karihaloo 1995.)

The displacement of the cohesive crack faces $w(s)$ (representing the FPZ) can be expressed as the following singular integral equation,

$$
\int_{0}^{\ell_{p}} g(s, t)\left[\sigma(s)-\sigma_{0}\left(\ell_{p}-s\right)\right] \mathrm{d} s=-w(t)
$$

The finite tensile strength of concrete requires that SIF vanish at the FPZ tip. This in turn requires that the faces of FPZ close smoothly, i.e.

$$
\int_{0}^{\ell_{p}} k(s)\left[\sigma(s)-\sigma_{0}\left(\ell_{p}-s\right)\right] \mathrm{d} s=0
$$

The weight functions $g(s, t)$ and $k(s)$ are the respective crack face opening displacement (COD) at the location $t$ and the SIF at the crack tip of a single edge cracked specimen of finite size due to a pair of unit normal forces at $s$ on the crack faces (figure 4). These have been derived by Xiao \& Karihaloo (2002)

$$
\begin{aligned}
g(s, t) & =-\frac{4}{\pi E^{\prime}} \ln \left|\frac{\sqrt{s}-\sqrt{t}}{\sqrt{s}+\sqrt{t}}\right|+\frac{8}{E^{\prime}}\left[A_{1} \sqrt{t}-A_{3} t^{3 / 2}+A_{5} t^{5 / 2},\right] \\
k(s) & =(2 / \pi s)^{1 / 2}+A_{1} \sqrt{2 \pi},
\end{aligned}
$$


where $E^{\prime}=E$ for plane stress and $E^{\prime}=E /\left(1-v^{2}\right)$ for plane strain, with $E$ the Young modulus and $v$ the Poisson ratio. Accurate interpolation formulae for $A_{1}, A_{3}$ and $A_{5}$ have been given by Xiao \& Karihaloo (2002) which depend on the size and geometry of the body,

$$
A_{i}(\alpha, \gamma) / W^{-(i / 2)+1}=\sum_{j=0}^{4} f_{i j}(\alpha) \gamma^{j}, i=1,3,5,
$$

where $\alpha=a / W, \gamma=s / a$ and $a$ is the crack length.

The functions $f_{i j}(\alpha)$ are

$$
\begin{aligned}
& f_{14}(\alpha)=\left\{\begin{array}{l}
\left(34.606 \alpha^{3}-41.019 \alpha^{2}+20.223 \alpha-3.7408\right) /(1-2 \alpha)^{3}, \\
0,
\end{array}\right\}, \\
& f_{13}(\alpha)=\left\{\begin{array}{l}
\left(-13.817 \alpha^{3}+27.952 \alpha^{2}-21.109 \alpha-4.8557\right) /(1-2 \alpha)^{3}, \\
0,
\end{array}\right\}, \\
& f_{12}(\alpha)=\left\{\begin{array}{l}
\left(20.791 \alpha^{3}-13.601 \alpha^{2}+11.745 \alpha-3.9004\right) /(1-2 \alpha)^{2}, \\
-3.2167 \alpha^{3}+3.22 \alpha^{2}-0.7748 \alpha-1.2004,
\end{array}\right\}, \\
& f_{11}(\alpha)=\left\{\begin{array}{l}
\left(16.38 \alpha^{3}-14.453 \alpha^{2}+0.0025 \alpha+1.378\right) /(1-2 \alpha)^{2}, \\
127.17 \alpha^{3}-157.39 \alpha^{2}+73.588 \alpha-9.5209,
\end{array}\right\}, \\
& f_{10}(\alpha)=\left\{\begin{array}{l}
11.067 \alpha^{3}-5.29 \alpha^{2}+0.9753 \alpha+0.0354, \\
\left(-6.3283 \alpha^{3}+11.659 \alpha^{2}-6.1031 \alpha+1.0268\right) / \alpha^{2},
\end{array}\right\}, \\
& f_{54}(\alpha)=\left\{\begin{array}{l}
\left(0.0213 \alpha^{3}-0.119 \alpha^{2}+0.0904 \alpha-0.0185\right) /(1-2.45 \alpha)^{4} \alpha^{3}, \\
0,
\end{array}\right\}, \\
& f_{34}(\alpha)=\left\{\begin{array}{l}
\left(2.4861 \alpha^{3}-1.6212 \alpha^{2}-0.0339 \alpha+0.1139\right) /(1-2.45 \alpha)^{3} \alpha^{3 / 2}, \\
0,
\end{array}\right\}, \\
& f_{32}(\alpha)=\left\{\begin{array}{l}
\left(1.9089 \alpha^{3}-3.0813 \alpha^{2}+1.8375 \alpha-0.3641\right) /(1-2.45 \alpha)^{3} \alpha^{3 / 2}, \\
7.2667 \alpha^{3}-10.215 \alpha^{2}+6.8468 \alpha-0.8862, \\
0,
\end{array}\right\}, \\
& \left.f_{33}(\alpha)=\left\{\begin{array}{l}
\left(251.04 \alpha^{3}-234.57 \alpha^{2}+75.35 \alpha-8.7819\right) /(1-2 \alpha)^{2}, \\
-607.25 \alpha^{3}+811.68 \alpha^{2}-376.24 \alpha+56.809,
\end{array}\right\}, 2 \alpha\right) 3 \alpha^{3 / 2},
\end{aligned}
$$




$$
\begin{aligned}
& f_{53}(\alpha)=\left\{\begin{array}{l}
\left(-0.3963 \alpha^{3}+0.5465 \alpha^{2}-0.2519 \alpha+0.0388\right) /(1-2.01 \alpha)^{6} \alpha^{3}, \\
0,
\end{array}\right\}, \\
& f_{52}(\alpha)=\left\{\begin{array}{l}
\left(19.996 \alpha^{3}-17.901 \alpha^{2}+5.595 \alpha-0.6524\right) /(1-2 \alpha)^{3} \alpha^{2}, \\
129.03 \alpha^{3}-204.66 \alpha^{2}+108.13 \alpha-17.662,
\end{array}\right\}, \\
& f_{51}(\alpha)=\left\{\begin{array}{l}
\left(-143.59 \alpha^{3}+142.36 \alpha^{2}-47.318 \alpha+5.3329\right) /(1-2 \alpha)^{3} \alpha^{2}, \\
\left(-187.17 \alpha^{3}+264.43 \alpha^{2}-130.17 \alpha+21.167\right) /(1-\alpha),
\end{array}\right\}, \\
& f_{50}(\alpha)=\left\{\begin{array}{l}
1.1667 \alpha^{3}+4.045 \alpha^{2}-3.0112 \alpha+0.3928, \\
-1.0667 \alpha^{3}-5.335 \alpha^{2}+4.4722 \alpha-0.9568,
\end{array}\right\} .
\end{aligned}
$$

In each of the above expressions except $f_{53}(\alpha)$, the first entry is for $\alpha<0.4$ and the second for $\alpha \geq 0.4$. In the expression of $f_{53}(\alpha)$ the first entry is for $\alpha<0.5$ and the second for $\alpha \geq 0.5$.

In the earlier formulation (Karihaloo 1999), the following crucial approximations were made: (i) Only the first terms in the weight functions (7) and (8) were used. These terms correspond to a semi-infinite crack in an infinite body and not the finite TPB beam considered; (ii) With this approximation, the two singular integral equations (5) and (6) were solved analytically in an indirect manner. The COD $w(s)$ in the FPZ $0 \leq s \leq \ell_{p}$ was approximated by a polynomial in $s$, and $\sigma(s)$ and $l_{p}$ were solved analytically from (5) and (6) for prescribed $\sigma_{r}$ (i.e. $\left.K_{I}\right)$. The distance $s$ was then eliminated from the assumed $w(s)$ and the calculated $\sigma(s)$ to establish the tension softening relationship $\sigma(w)$.

As a result of the above approximations, the $\sigma(w)$ relationship so obtained became dependent on the geometry of the structure and external loading (i.e. on $\sigma_{r}$ and $a_{3}$ ), and no longer reflected a true material property, as it should. Additionally, the nominal strength formula (2) predicted an arbitrary lower limit on the structural size $W>B_{2}$ in order for the formula to have a physical meaning.

The above exact formulation has overcome these drawbacks. However, it is now necessary to prescribe the actual $\sigma(w)$ diagram for the concrete used for making the TPB beams and to solve the singular integral equations (5) and (6) numerically.

For the HSC reported above, the following tension softening diagram (figure 5) seems most appropriate

$$
w / w_{c}=\left[\left(\sigma / f_{t}\right)^{-1 / 3}-1\right] /\left[\left(s_{01} / f_{t}\right)^{-1 / 3}-1\right],
$$

where $w_{c}$ is the critical crack opening, and $s_{01}$ is the residual cohesive stress at the original crack tip when it begins to propagate. This is required to ensure convergence of the numerical integration scheme.

From the definition of the specific fracture energy $G_{F}$ according to the fictitious crack model,

$$
G_{F}=\int_{f_{t}}^{s_{01}} w(\sigma) \mathrm{d} \sigma,
$$

it follows that

$$
\frac{G_{F}}{w_{c} f_{t}}=\frac{-1.5\left(s_{01} / f_{t}\right)^{2 / 3}+s_{01} / f_{t}}{\left(s_{01} / f_{t}\right)^{-1 / 3}-1}
$$




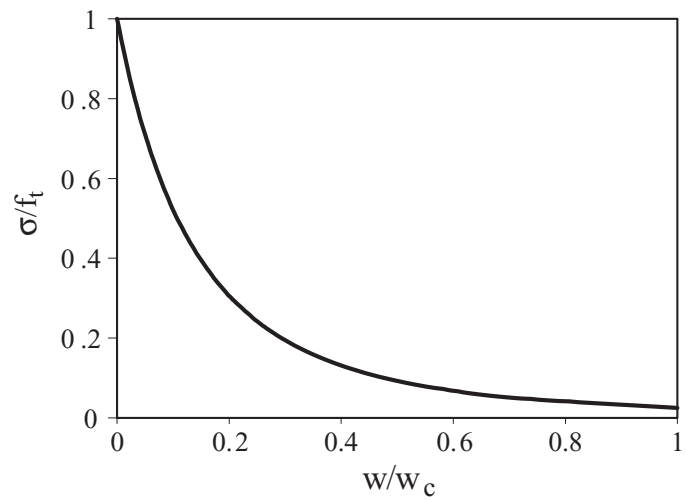

Figure 5. Tension softening diagram.

If we assume $s_{01}=0.025 f_{t}$, then $w_{c}$ can be calculated from (12) knowing $G_{F}=44.5 \mathrm{~J} / \mathrm{m}^{2}$ and $f_{t}=7.40 \mathrm{MPa}$ for the HSC used by Karihaloo \& Abdalla (2001)

$$
G_{F} / w_{c} f_{t}=0.164 \Rightarrow w_{c}=0.03683 \mathrm{~mm} .
$$

The results are shown in figure 6 .

\section{Unnotched HSC beams}

A limited number of unnotched HSC beams were also tested in three-point bending. The depth of these beams covered a wide range, from 50 to $400 \mathrm{~mm}$. All beams were $100 \mathrm{~mm}$ wide. The mean failure loads and nominal strength values are given in table 2 .

The mean nominal strength is plotted against the beam depth in figure 7 , together with the line of best fit. The odd values for depths 75 and $300 \mathrm{~mm}$ are presumably due to just one specimen being available for testing. The trend is, however, very clear - the strength reaches the asymptotic value for large sizes from above.

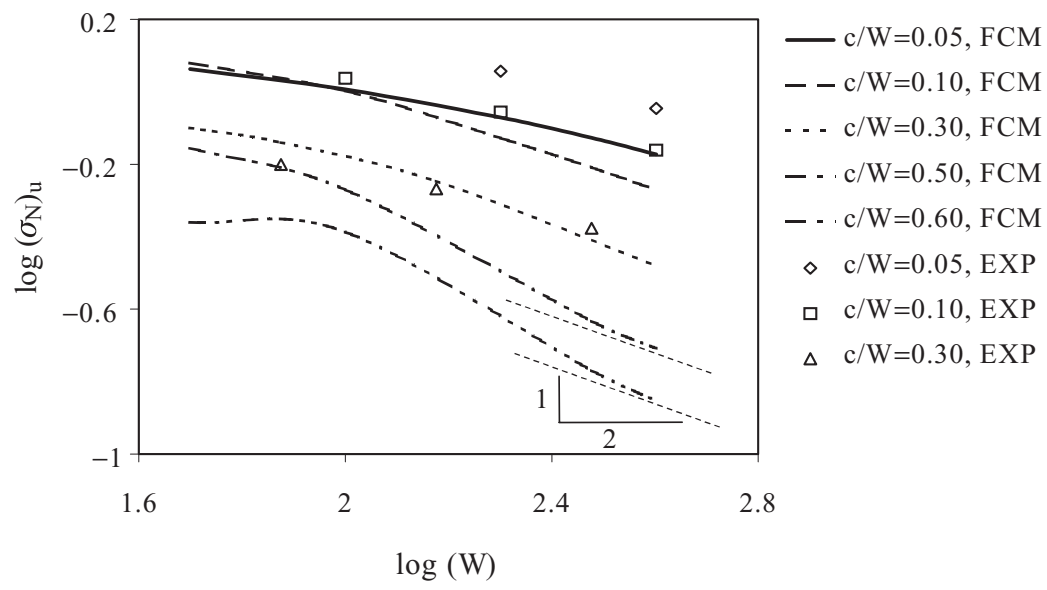

Figure 6. Variation of the nominal strength $\left(\sigma_{N}\right)_{u}\left(=\sigma_{u} / 6\right)$ in MPa with depth W in mm for a TPB with various $\alpha$ and $S / W=4$ on a $\log -\log$ plot. 
Table 2. Failure loads for unnotched HSC beams.

\begin{tabular}{lcc}
\hline $\begin{array}{l}\mathrm{W} \\
(\mathrm{mm})\end{array}$ & $\begin{array}{c}\text { Mean } \\
P_{u}(\mathrm{kN})\end{array}$ & $\begin{array}{c}\text { Mean } \\
\left(\sigma_{N}\right)_{u}(\mathrm{MPa})\end{array}$ \\
\hline 50 & 9.40 & 1.88 \\
75 & 11.90 & 1.59 \\
100 & 16.40 & 1.64 \\
150 & 24.45 & 1.63 \\
200 & 30.40 & 1.52 \\
300 & 41.53 & 1.38 \\
400 & 56.50 & 1.41 \\
\hline
\end{tabular}

The measured values are compared in figure 8 with the predictions of the multifractal scaling (MFSL) formula (3) due to Carpinteri. The constants $A_{3}$ and $B_{3}$ in this formula are calculated by a standard regression approach. Formula (3) but with the exponent 1/2 replaced by 1 also gives the nominal strength at crack initiation as obtained by Bažant (1997). The constants $A_{3}$ and $B_{3}$ are also determined by linear regression. The constant $A_{3}$ represents the nominal strength for an infinitely large beam, whereas the constant $B_{3}$ is related to the thickness of the so-called "boundary layer" of cracking. The Bažant modification of (3), denoted SEL, is also shown on figure 8 .

For unnotched HSC beams, Carpinteri's formula (3) consistently gives better predictions than does Bažant's formula, although the difference in the predictions of the two is rather small.

\section{Concluding remarks}

In conclusion, it must be pointed out that the above work did not contribute to a resolution of the controversy raging in the literature. In particular, it did not shed any light on why the strength of notched beams approaches the asymptotic limit for large sizes from below, whereas that of unnotched beams approaches it from above. However, preliminary results from the new theoretical work briefly described in $\S 3$ above show that there is a change in

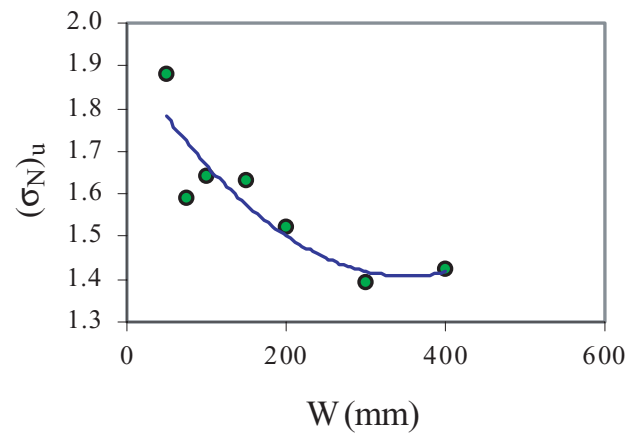

Figure 7. Variation in nominal failure strength with beam depth for unnotched HSC beams.

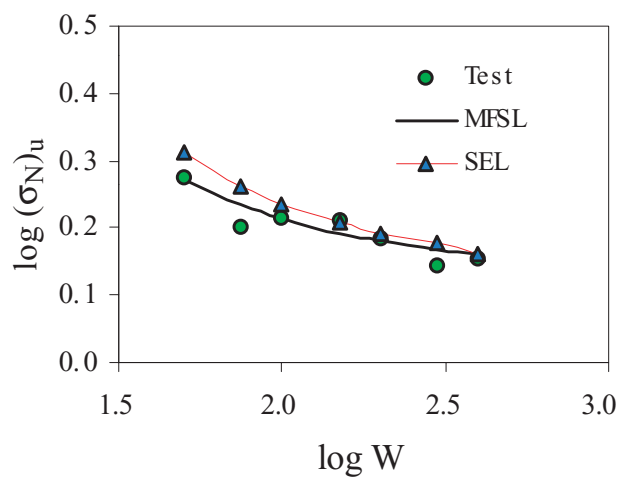

Figure 8. Size effect plots for unnotched TPB beams. 
the curvature of the size effect plot (i.e. $\log \left(\sigma_{N}\right)_{u}$ vs $\log W$ ) as the notch to depth ratio goes towards zero. These results are most encouraging and, if confirmed by a detailed analysis, will be reported in future communications.

Financial support from the EPSRC under grant no. GR/M78106 is gratefully acknowledged.

\section{References}

Bažant Z P 1984 Size effect in blunt fracture: Concrete, rock, metal. J. Eng. Mech., Am. Soc. Civil Eng. 100: 518-535

Bažant Z P 1997 Scaling of quasi-brittle fracture: Asymptotic analysis. Int J. Fracture 83: 19-40

Carpinteri A 1994a Fractal nature of material microstructure and size effects on apparent mechanical properties. Mech. Mater. 18: 89-101

Carpinteri A 1994b Scaling laws and renormalization groups for strength and toughness of disordered materials. Int. J. Solids Struct. 31: 291-302

Carpinteri A, Ferro G 1994 Size effect on tensile properties: A unified explanation based on disorder and fractality of concrete microstructure. Mater. Struct. 27: 513-571

Karihaloo B L 1995 Fracture mechanics and structural concrete (Harlow, Essex: Addison Wesley, Longman) pp 151-165

Karihaloo B L 1999 Size effect in shallow and deep notched quasi-brittle structures. Int. J. Fracture 95: 379-390

Karihaloo B L, Abdalla H M 2001 Size effect in hardened cement paste and high strength concrete. In Fracture mechanics of concrete structures (eds) de Borst et al (Lisse: Balkema) vol.2, pp 643-649

Karihaloo B L, Xiao Q Z 2001a Higher order terms of the crack tip asymptotic field for a notched three-point bend beam. Int. J. Fracture 112: 111-128

Karihaloo B L, Xiao Q Z 2001b Higher order terms of the crack tip asymptotic field for a wedgesplitting specimen. Int. J. Fracture 112: 129-137

Xiao Q Z, Karihaloo B L 2002 Approximate weight functions for singular and higher order terms of an edge crack in a finite plate. Engng. Fract. Mech. 69: 959-981 